\title{
Design and Evaluation of the LOPES Exoskeleton Robot for Interactive Gait Rehabilitation
}

\author{
Jan F. Veneman, Rik Kruidhof, Edsko E. G. Hekman, Ralf Ekkelenkamp, Edwin H. F. Van Asseldonk, and \\ Herman van der Kooij
}

\begin{abstract}
This paper introduces a newly developed gait rehabilitation device. The device, called LOPES, combines a freely translatable and 2-D-actuated pelvis segment with a leg exoskeleton containing three actuated rotational joints: two at the hip and one at the knee. The joints are impedance controlled to allow bidirectional mechanical interaction between the robot and the training subject. Evaluation measurements show that the device allows both a "patient-in-charge" and "robot-in-charge" mode, in which the robot is controlled either to follow or to guide a patient, respectively. Electromyography (EMG) measurements (one subject) on eight important leg muscles, show that free walking in the device strongly resembles free treadmill walking; an indication that the device can offer task-specific gait training. The possibilities and limitations to using the device as gait measurement tool are also shown at the moment position measurements are not accurate enough for inverse-dynamical gait analysis.
\end{abstract}

Index Terms-Body-weight supported treadmill training, exoskeleton robot, gait training device.

\section{INTRODUCTION}

A RECENT development in robotics is the design of robots for the mechanization of physical therapy, usually referred to as robotic (neuro-)rehabilitation or robot-mediated (or -aided) therapy [3]-[6]. These robots replace the physical training effort of a therapist. This may be useful in cases where a therapist's effort is very intensive leading to limitations in availability or even injuries. In the general setting of these robotic systems, a therapist is still responsible for the nonphysical interaction and observation of the patient by maintaining a supervisory role of the training, while the robot carries out the actual physical interaction with the patient. Several groups are currently developing robots for "arm training," as well as for "gait training," which is the focus of this paper.

Current automated gait trainers, such as the Lokomat (Hocoma, AG, Volketswil, Switzerland) [7], the pneumatically operated gait orthosis and the pelvic assist manipulator (known as POGO and PAM, respectively; not commercially available)

Manuscript received September 15, 2006; revised March 5, 2007; accepted May 3, 2007. This work was supported by the Institute for Biomedical Technology. The work of H. van der Kooij was supported by the Netherlands Organisation of Scientific research (Vernieuwings-impuls 2001, 016027011). This paper was presented in part at the 28th Annual International Conference of the IEEE Engineering in Medicine and Biology Society, New York, August/ September 2006.

The authors are with the Institute for Biomedical Technology (BMTI), University of Twente, 7500 EA Enschede, The Netherlands (e-mail: h.vanderkooij@utwente.nl).

Digital Object Identifier 10.1109/TNSRE.2007.903919
[8], the GaitTrainer (Reha-Stim, Berlin, Germany) [9], the Haptic Walker (not commercially available) [10], and the AutoAmbulator (HealthSouth Cooperation), are usually unable to fully adapt their movements to the activity of the patient. Some devices are not able to assist all possible leg movements, but for example only foot movement.

This paper describes the design and performance of the gait rehabilitation robot LOPES (lower extremity powered exoskeleton). Design choices will be motivated, the prototype presented, and its performance demonstrated.

\section{DESIGN CRITERIA}

At the moment, determinants of successful gait training are largely unknown, although intensity [11], self-initiative [12] and task specificity [13], [14] seem to be important. This is also reflected in current research on robotic neurorehabilitation [8], [15], [16]. These facts call for a device that allows near-tonormal free walking and that allows providing a wide range of possible content of training and supportive actions, while safety (for both patient and therapist) is assured at any time.

In order to satisfy these requirements, a robot, in the first place, should allow for a "patient-in-charge" mode where healthy subjects are able to walk unconstrained by the device. This concerns the choice of degrees-of-freedom (DOF) and the quality of low impedance control. The patient-in-charge mode can be considered as the "ideal" end situation of training, resembling how a patient would perform outside the device. It assures that foot-sole sensory input (during free walking in the device) will be near to normal. The availability of this mode is important for patients with a nonparetic side, which should not be disturbed during its operation. Second, the robot should allow for a "robot-in-charge" mode, whereby the robot is able to move a passive subject in a gait-like motion. From design viewpoint, this concerns both torque and speed limitations of the actuation on the actuated DOFs. Thus, the robot-in-charge-mode is a position control mode, assuring that the robot can provide all selective/partial assistance to keep an inactive patient walking. Third, the robot should allow for a "therapist-in-charge" mode whereby any required therapeutic interventions can be programmed. Note, that this mode demands the ability of the actuators to act as pure force sources, leading to implementation of the desired impedance patterns. In stark contrast to fully automated gait trainers, the proposed approach allows a therapist to stay in control of the provided therapy and to implement several training strategies. 




Fig. 1. Basic outline of an impedance controlled device, applied on robotic therapy. Here, the connections between device and patient are taken as a part of the patient impedance, so that the device can be considered rigidly connected to the "patient". The " $\mathrm{x}$ " indicates position and " $\mathrm{F}$ " indicates force.

\section{PRototyPe Design}

\section{A. Impedance Controlled Exoskeleton}

Exoskeleton: In order to allow for corrective forces or torques to the legs of a patient, a so-called exoskeleton type robot was designed. The robot moves in parallel to the skeleton of the patient, so that no additional DOF or motion ranges are needed to follow patient motions. As the exoskeleton-joint-motions of the robot directly correspond with the motions of a patient's joints, it is relatively easy to implement mechanical safety limits to motion- and torque-ranges into the exoskeleton structure. However, a few shortcomings of an exoskeleton include the need to accurately align joints and the need for high torques. The latter may be necessary for interventions that could actually be carried out by smaller end-effector forces.

The exoskeleton as a whole is physically connected to an actuated support located at pelvis height. The virtue of this type of setup allows for weight-compensation of the exoskeleton and for applying external corrective forces to the pelvis of the patient, instead of only muscle-like internal torques at leg level. This approach leads to additional possibilities for interventions. Thus, the LOPES robot is a combination of an exoskeleton robot for the legs and an externally supporting end-effector robot for the pelvis.

Impedance Controlled: Impedance control, as opposed to admittance control, was selected as a basic interaction control outline for the exoskeleton (see Fig. 1). Impedance control implies that the interaction control is based on position sensing combined with force actuation [2], [17].

While designing the robot, the choice of impedance control implies that the moving parts of the construction must be lightweight and that the actuators are "pure" force sources. The use of impedance control for an exoskeleton is advocated in [2] and [18].

Training interventions will be programmed using virtual model control, an implementation of impedance control based on the definition of virtual dynamic components, e.g., virtual springs [19].

DOF: An optimal set of DOFs was chosen in order to allow for a subject to walk normally and safely in the device. After studying the literature on gait, and analyzing the experiences with existing devices and tests with a first prototype, a total number of eight actuated DOFs (two for the horizontal pelvis translation and three rotational joints per leg) were considered to be sufficient. One DOF (the vertical motion of the pelvis) was passively weight compensated by means of an ideal spring-
TABLE I

ACTUATED, FREE, AND BLOCKED DOF OF THE LOPES EXOSKELETON

\begin{tabular}{|c|c|c|c|c|}
\hline \multirow{2}{*}{ DoF } & \multirow{2}{*}{$\begin{array}{c}\text { Human } \\
\text { possibility }\end{array}$} & \multicolumn{3}{|c|}{ Robot design } \\
\hline & & Actuated & Free & Blocked \\
\hline $\begin{array}{l}\text { Pelvis to } \\
\text { fixed } \\
\text { world }\end{array}$ & $\begin{array}{c}3 \text { rotations } \\
3 \text { translations }\end{array}$ & $\begin{array}{c}\text { Left/Right } \\
\text { Forwd/backwd }\end{array}$ & Up/down & $\begin{array}{l}\text { All rotations } \\
\text { except for play }\end{array}$ \\
\hline Hip & 3 rotations & $\begin{array}{l}\text { Ad-/Abduction } \\
\text { Flex-/extension }\end{array}$ & - & $\begin{array}{c}\text { Exo/endo- } \\
\text { rotation except } \\
\text { for play } \\
\end{array}$ \\
\hline Knee & $\begin{array}{l}1 \text { rotation } \\
\text { (sagittal) }\end{array}$ & Flex-/extension & Ad-/Abduction ${ }^{1}$ & - \\
\hline Ankle & 3 rotations $^{2}$ & - & All motions & - \\
\hline
\end{tabular}

${ }^{1}$ The knee abduction is not a human possibility, but was left free for constructional reasons, to not lead forces through the knee joint.

${ }^{2}$ The ankle is a complex joint, where the axes of motion are not simply the three Euclidian axes.

mechanism, and left free to move unactuated within designed limits. All eight DOFs not only allow the exoskeleton to make a forward stepping motion (as provided by the Lokomat and the AutoAmbulator), but also maintain the fundamental instability of a standing or walking human. As such, balance control still has to be achieved when walking in the device, either by the human or (when necessary) by the robot, and is widely recognized as an important aspect of gait training [8], [20].

Table I describes which DOFs are possible for a human being, which of these are actuated in the robot, which are left free, and which are blocked.

The reason to omit an actuated robotic ankle joint was that it is not necessary to provide an external "ankle push-off" in the device in order to walk safely. Also, it is possibly painful to apply substantial torque to the feet, at least without using an individually fit-to-size foot-interface. The patient's forward progression can be assured by the treadmill together with the pelvis actuation. For patient safety, the only necessary ankle function is to assure enough foot clearance during swing. This can be realized with simpler means such as using elastic straps, or a passive orthosis. Of course the robot should allow a recovering patient to generate an ankle push-off during rehabilitation; this not only involves the availability of the natural ankle's DOFs, but also available pelvis translations, so the body can actually be accelerated by a push-off.

In case that an ankle push-off would be externally provided during training, it is important that the device exerts its force through the foot and not directly to the floor as this would affect the sensed ground reaction forces, disturbing the patient's normal afferent input. However, what could be useful for training purposes, is the possibility to provide small torques around the ankle in order to "suggest" when the patient should "push-off." Devices like the pneumatically driven ankle orthosis [21] or the Anklebot [22] are examples of what is possible. These or similar devices can be added to the LOPES if ankle actuation appears to be crucial from a clinical point of view.

Table II presents the peak torques and ranges of motion that where chosen as specifications for the actuated DOFs. Torques 
TABLE II

SPECIFICATIONS FOR THE ACTUATED DOF OF THE LOPES EXOSKELETON

\begin{tabular}{l||l|l|l}
$\begin{array}{l}\text { Degree of } \\
\text { freedom }\end{array}$ & $\begin{array}{l}\text { Range of } \\
\text { motion }\end{array}$ & $\begin{array}{l}\text { Maximum } \\
\text { torque/force }\end{array}$ & $\begin{array}{l}\text { Maximum } \\
\text { speed }\end{array}$ \\
\hline \hline Hip abduction & $+15^{\circ} /-15^{\circ}$ & $30 \mathrm{Nm}$ & $1 \mathrm{rad} / \mathrm{s}$ \\
\hline Hip flexion & $+60^{\circ} /-30^{\circ}$ & $65 \mathrm{Nm}$ & $2 \mathrm{rad} / \mathrm{s}$ \\
\hline Knee flexion & $+0^{\circ} /-90^{\circ}$ & $65 \mathrm{Nm}$ & $5 \mathrm{rad} / \mathrm{s}$ \\
\hline Sideways & $+0.30 \mathrm{~m} /-0.30 \mathrm{~m}$ & $250 \mathrm{~N}$ & $0.5 \mathrm{~m} / \mathrm{s}$ \\
\hline For-/back-ward & $+0.35 \mathrm{~m} /-0.35 \mathrm{~m}$ & $200 \mathrm{~N}$ & $0.5 \mathrm{~m} / \mathrm{s}$ \\
\hline Up/down & $+0.10 \mathrm{~m} /-0.10 \mathrm{~m}$ & $\begin{array}{l}\text { Passively weight } \\
\text { compensated }\end{array}$ & -
\end{tabular}
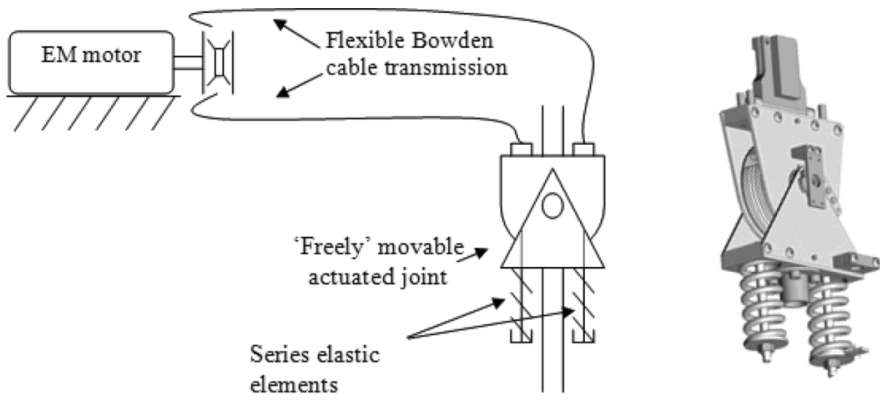

Fig. 2. Schematic and graphic representation of the used joint actuators in the exoskeleton. Bowden cable driven series elastic actuators [2].

and forces were chosen based on measured values of joint torques in the "slow walking cycle" [23], and on estimates and measurements of forces that therapists apply during conventional gait training [24]. The presented torque/speed values are in fact the peak values that appear in the speed-torque curve while walking, where both maxima do not appear at the same time. However, using these values as general demands overestimates the nominal power that is needed. Thus, due to uncertainties in the measured values and the need for an extra margin for safety, the overestimated values are used in the design.

\section{B. Realization of the LOPES Prototype}

Based on the aforementioned section, we designed an exoskeleton with three rotational joints per leg: two at the hip (abduction-4 and flexion-5, for numbers, see Fig. 3 ) and one at the knee (flexion-6). The physical hip abduction joint is placed behind the patient, where the position of the hip abduction axis relative to the hip flexion axis is fixed. The position of both abduction axes relative to each other (pelvis width), and the position of the hip axis relative to the knee axis (upper leg length) are adjustable, and are adjusted to suit the dimensions of each patient. The joints of the robot are actuated with Bowden-cable driven series elastic actuators (see Fig. 2). This concept was chosen in order to implement low weight "pure" force sources. The concept, construction, and functionality of these joints are described extensively in [2]. This type of actuator was used for all rotary joints, and the sideways pelvis translation is equipped with a linear version of the same actuation principle. Finally, the forwards and backwards motion is driven by an open-loop force-controllable linear actuator.

The prototype uses Kollmorgen/Danaher AKM22C servomotors, with a maximum speed of $8000 \mathrm{rpm} ; 567 \mathrm{~W}$ rated



Fig. 3. DOF of the pelvis and leg segments of the LOPES gait rehabilitation robot: (1) forward linear guide, (2) sideways linear guide, (3) parallelogram for vertical motion, (4) hip frontal rotation, (5) hip sagittal rotation, and (6) knee sagittal rotation. The two horizontal motions (1) and (2) and the hip frontal rotation (6) are optionally blocked in the experiments. Except for (3) are all mentioned DOFs actuated. (A) indicated the height adjustability of the support frame.

power, and a continuous torque of $0.87 \mathrm{Nm}$ and a peak torque of $2.73 \mathrm{Nm}$. This motor is used in combination with a Neugart Planetary gearhead that reduces speed with a ratio of $64: 1$ where these sets are used for all (6) rotary DOFs. For the sideways motion, a Berger Lahr SER3910 is used with a maximum speed of $6000 \mathrm{rpm}$, a peak torque of $2.2 \mathrm{Nm}$, and a rated power of $690 \mathrm{~W}$. This motor is used in combination with a Neugart Planetary gear head with a reduction of $8: 1$. For the forward/backward motion, a Linmot P01-37 $\times 240$ linear actuator is used with a rated power of $250 \mathrm{~W}$ and a peak force of $204 \mathrm{~N}$. The linear springs applied in the flexion joints (hip and knee) have a stiffness of $35.1 \mathrm{kN} / \mathrm{m}$ each; those applied in the hip abduction joints have a stiffness of $57.2 \mathrm{kN} / \mathrm{m}$; and the springs applied for the left right actuation have a stiffness of $3.98 \mathrm{kN} / \mathrm{m}$.

The change in spring length, which is used as force measurement in the actuators, is measured with linear slider potentiometers (where the sliders are connected to a spring). The construction for connecting this exoskeleton to the fixed world consists of (for explanation of the numbers, see Fig. 3).

1) A height-adjustable frame to match the length of the patient (A), this height is fixed during training, and needs to be adjusted at the beginning of a training session.

2) Two sets of perpendicularly placed parallel bars with carriages for the forward/backward (1) and the sideways (2) motion; double bars are used to translate load torques into forces.

3) A parallelogram with bearings (3) and weight compensation to allow limited vertical motion during operation. The weight compensation is realized with an "ideal spring" mechanism.

This construction is then placed over a treadmill. The motors that drive the robot joints are placed at the back of the construction, and connected to the robot joints by two Bowden cables 



Fig. 4. Photographic impression of the prototype of LOPES. Two Bowden cables per joint that transmit the power from motors to the robot joints are visible for several joints. Right-most pictures show how a person is connected to the device. In the left picture, above the back plate, the connector for the cushion is visible.

per actuated DOF. A photographic impression of the resulting total construction of the LOPES is shown in Fig. 4.

\section{Evaluation of Performance}

\section{A. Evaluation Methods}

In order to evaluate the performance of the design and avoid extensive subject or patient tests, several hardware tests were performed based on the demands stated in Section II. To prove the functionality of the "patient-in-charge" mode, the torque responses to imposed motions were measured. In all cases, the joints were operated in zero-torque control. First, the force response of a single disconnected knee-joint was measured. Second, a leg was constructed of two joints: hip and knee, and leg segments. This leg was placed in a test setup where controlled motions could be imposed at the ankle. The Cartesian impedance at the ankle, the end-effector, was then determined by measuring the force response to an imposed multisine motion in the range $0.1-4 \mathrm{~Hz}$ with uniform power distribution. Third, in the full prototype, the peak force responses to hand-imposed motions on all eight DOFs were measured. This last measurement was carried out per separate joint, while fixating the other joints. Motions were exerted by hand, via the force sensor, yielding realistic motion speeds. The motions had a frequency-range of $1-3 \mathrm{~Hz}$ and an amplitude of about $30^{\circ}$ for knee at $1 \mathrm{~Hz}$ down to $10^{\circ}$ at $3 \mathrm{~Hz}$. These values were $20^{\circ}$ and $5^{\circ}$, respectively, for the hip flexion and about $10^{\circ}$ and $5^{\circ}$ for the hip abduction. Translations showed amplitudes of 10 and $2 \mathrm{~cm}$ for 1 and $3 \mathrm{~Hz}$, respectively. For the measurement of knee and hip flexion torques, the sensor was mounted at ankle height, for the measurement of hip abduction at the knee, and for the measurement of the pelvis motions in the middle of the back plate, where during operation the low back cushion is attached (see Fig. 4). From the force vector, and the known location of the force sensor, the torque around the joint is calculated.

The ultimate test of the "patient in charge" mode is the comparison between free treadmill walking and walking in the LOPES while controlled to zero impedance. These tests have also been finished, but the findings are too extensive and will be published elsewhere. Preliminary results of electromyography (EMG) measurements of the activation patterns of eight major leg-muscles of one healthy young (age: 21) female subject at a walking speed $0.75 \mathrm{~m} / \mathrm{s}$ will be shown. This gives a good indication of changes that appear when walking in the device.

To evaluate the "robot-in-charge" mode achievable standstill peak forces on the robot joints are measured. In order to evaluate the "therapist-in-charge" mode the force bandwidth of the separate robot joints is measured, as this determines the achievable bandwidth of any impedance control. In both cases, all forces are measured with a 6-D force sensor (ATI-Mini45-SI-580-20) connected to the robot leg.

Finally, the orientation and position of the leg segments compared to the robot segments are measured as this is important both for accurate control of LOPES and for its use as a measurement/diagnosis device. For the human leg orientation measurements, we used a PTI-VZ4000 mocap system from PhoeniX Technologies. This system follows active, uniquely identifiable LEDs in 3-D space using a camera bar, where the motion of both robotic and human limbs (for one leg) are tracked. In order to track the "human leg," clusters comprised of four markers each are stuck onto the back of both the upper and the lower leg. It was necessary to place the camera on the left behind the subject due to limited visibility of leg markers caused by the mechanical construction.

During walking, the translations of the pelvis and the hip abduction/adduction were blocked, so that the walking could be considered purely sagittal - this way the rotation axes could be defined as global stationary axes. Finally, the hip and knee rotation of both human and robot were then tracked during walking and mutually compared. A step-cycle trajectory averaged over fifteen steps, averaged over ten unimpaired subjects (mean age 26) is presented as the result of this measurement. All subjects taking part in measurements provided informed consent prior to measurements.

\section{B. Evaluation Results}

Typical values for the resistive torque of the "Bowden cabledriven series elastic actuators" [2] when externally rotated were $0.2 \mathrm{Nm}$, for $1-\mathrm{Hz}$ imposed rotations to $0.7-\mathrm{Nm}$ peaks, for $4-\mathrm{Hz}$ rotations. These values were measured while controlling the joint to zero force, and with optimal Bowden cable courses. In this case, no additional inertia or weight was added to the joint, so solely the impedance of the actuator system and the friction of the joint were measured, not the inertial properties of the robot segments. 

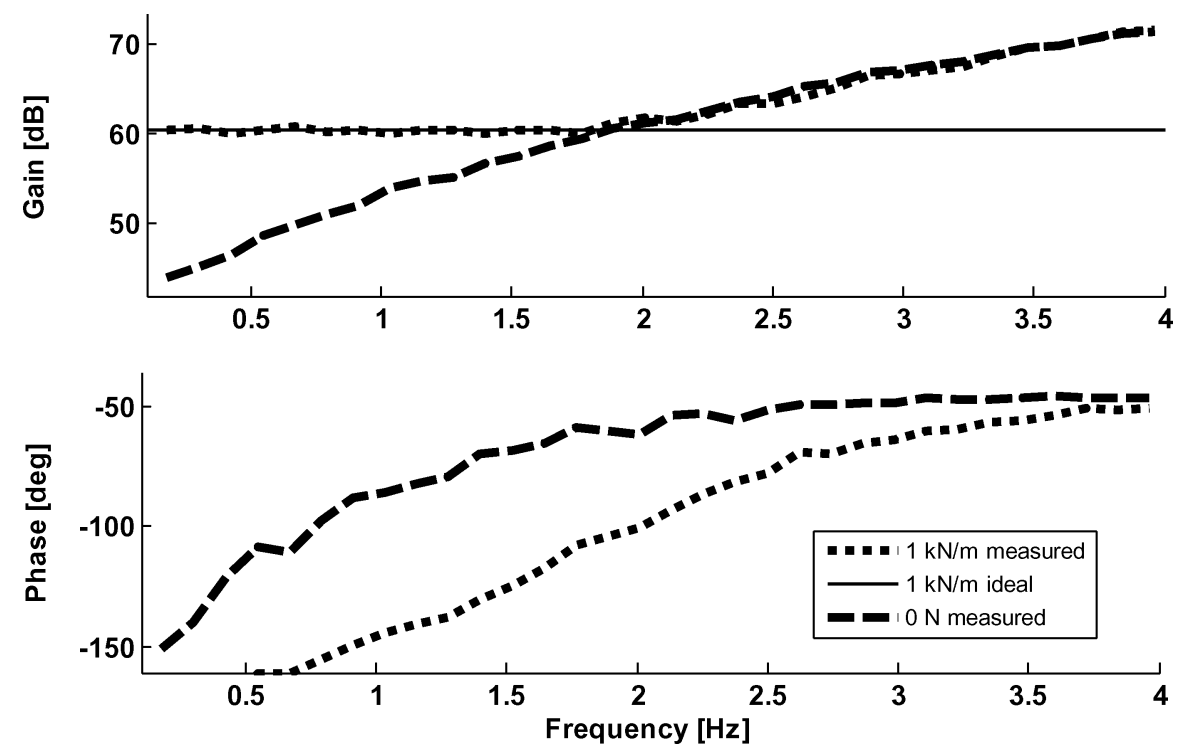

Fig. 5. Bode diagram of the measured Cartesian impedance at the ankle end effector of a leg with a hip and knee joint. The dB values of the force response to an imposed position multisine are presented. For comparison, a controlled stiffness of $1 \mathrm{kN} / \mathrm{m}$ is shown (ideal and measured). Zero impedance control shows the behavior of a damped mass.

TABLE III

MEASURED VALUES FOR RESISTIVE TORQUeS TO IMPOSED MOTIONS AND MAXIMALLY EXERTED TORQUES ON THE DOF OF THE LOPES EXOSKELETON

\begin{tabular}{l||l|l|l|l}
$\begin{array}{l}\text { Degree of } \\
\text { Freedom }\end{array}$ & $\begin{array}{l}\text { Actuator } \\
\text { Resistive } \\
\text { Torque or } \\
\text { Force }\end{array}$ & $\begin{array}{l}\text { 'Endpoint' } \\
\text { Resistive } \\
\text { Torque or } \\
\text { Force at } \sim 1 \\
\mathrm{~Hz}\end{array}$ & $\begin{array}{l}\text { 'Endpoint' } \\
\text { Resistive } \\
\text { Torque or } \\
\text { Force at } ~ \\
\mathrm{~Hz}\end{array}$ & $\begin{array}{l}\text { Maximum } \\
\text { exerted } \\
\text { Torque or } \\
\text { Force }\end{array}$ \\
\hline \hline Knee flexion & $<1 \mathrm{Nm}$ & $1.75 \mathrm{Nm}$ & $4 \mathrm{Nm}$ & $50 \mathrm{Nm}^{*}$ \\
\hline $\begin{array}{l}\text { Hip flexion } \\
\text { Hip } \\
\text { abduction }\end{array}$ & $<1 \mathrm{Nm}$ & $5 \mathrm{Nm}$ & $25 \mathrm{Nm}$ & $50 \mathrm{Nm}^{*}$ \\
\hline $\begin{array}{l}\text { Sideways } \\
\text { For-/back- } \\
\text { ward }\end{array}$ & $<5 \mathrm{~N}$ & $5 \mathrm{Nm}$ & $12 \mathrm{Nm}$ & $30 \mathrm{Nm}$ \\
\hline
\end{tabular}

* The peak torques of $50 \mathrm{Nm}$ mentioned for knee and hip flexion were actually measured. Due to the friction based force transmission, higher torques were not feasible yet, as some slip at the joint cable discs appeared. This will be fixed by redesign of the connection of the cables to the joints in such fashion that no slip can occur. The motors provide a continuous torque of max. $65 \mathrm{Nm}$ at the actual joint output axis, with a peak torque of over $100 \mathrm{Nm}$, for short intervals ( $<1$ second).

In the test setup with a leg comprised of a hip and knee joint, an accurate impedance characteristic of the Cartesian impedance at the ankle "end-effector" was measured (see Fig. 5). For easier interpretation, the zero force/impedance control measurement is accompanied by a measurement where the $1 \mathrm{kN} / \mathrm{m}$ stiffness is controlled. The Bode diagram shows that in case of the zero impedance control, a damped mass is sensed. With a controlled stiffness, the same mass is sensed at higher frequencies, while a stiffness together with a slight phase shift is measured at lower frequencies. Achievable bandwidths in the final prototype are about $4 \mathrm{~Hz}$ for the full force range $(65 \mathrm{Nm})$ up to $12 \mathrm{~Hz}$ for smaller forces $(<10 \mathrm{Nm})$.

Measuring the "endpoint" resistive torques in the final prototype yielded the values shown in Table III. For interpretation of the difference between the actuator resistive torque and the "endpoint" resistive torque, the component of this resistance
TABLE IV

INERTIAL TORQues at SPECIFIC MOTION PROFILES (CALCUlated INERTIAL FORCE RESPONSES TO EXERTED SINUSOID MOTION)

\begin{tabular}{l|l|l} 
& Whole leg (hip joint) & Lower leg (knee joint) \\
\hline $1 \mathrm{~Hz}$ sine & $13 \mathrm{Nm}(20 \mathrm{deg})$ & $1.9 \mathrm{Nm}(30 \mathrm{deg})$ \\
\hline $3 \mathrm{~Hz}$ sine & $29 \mathrm{Nm}(5 \mathrm{deg})$ & $5.7 \mathrm{Nm}(10 \mathrm{deg})$
\end{tabular}

caused by inertia alone are calculated. The results are based on the mass of a leg segment, $3 \mathrm{~kg}$ for the lower (including joint) and $6 \mathrm{~kg}$ for the whole leg. The centers of gravity are taken at the halfway points in each segment respectively $(40 \mathrm{~cm}$ from the hip for the whole leg and $17.5 \mathrm{~cm}$ from the knee for the lower leg). These values are approximate, as in practice they will change slightly due to the adaptations of segment lengths and connections to the patient. Finally, the masses are taken as point masses and the results of these calculations are shown in Table IV.

These values indicate the sizes of inertial torques appearing during walking, and show that the inertia of the construction explains most of the resistive torques that appear when moving the construction.

In order to show the effect of these torques on walking, the integrated EMG patterns of eight major leg muscles are compared for free treadmill walking and walking when connected to the LOPES controlled in zero impedance mode (Fig. 6). Analyzing Fig. 6, notice that the difference in patterns is small, especially when timing is considered, and that the Biceps Femoris shows an increase in EMG amplitude. This change takes place in the late swing, and can possibly be attributed to the increased leg mass. Also, the Adductor Longus and the Tibialis Anterior show slight but remarkable changes. The change in adductor activity was accompanied by a smaller step width. The change in Tibialis Anterior can also be explained by the extra mass, together with the need to assure sufficient foot clearance during swing.

The Glutues Medius shows a slight decrease in activity during late stance. This may be attributed to less active push-off, as the progression of the subject is assured by the moving treadmill and forces on the pelvis keeping the subject in place. 




compared to treadmill walking connected to the LOPES in zero impedance control mode ("patient in charge").

The peak forces and torques achieved on every DOF are presented in Table III, the right column. The bandwidth of feedback force control in the joints alone was measured at $11 \mathrm{~Hz}$ for the full force range (there $50 \mathrm{Nm}$ ) and $20 \mathrm{~Hz}$ for smaller forces $(<10 \mathrm{Nm})$ in an externally fixed joint, for optimal Bowden cable course; see [2] for the exact description of measurements and further nuances. Due to suboptimal cable courses in the prototype and of the inertia of the robot segments, the bandwidth of resulting force control at the "end-effector"-the ankle-is lower, down to about $2 \mathrm{~Hz}$ for larger forces in the ankle in motion, as can be seen in Fig. 5.
Fig. 7 shows the sagittal hip angle for one step cycle, averaged over steps and over subjects and the same information for the sagittal knee angle. The bigger differences for the hip angle can be explained by the fact that the cluster marker on the upper leg is positioned on top of the hamstrings, and this appears to cause extra motion (secondary rotations) of the cluster.

At certain moments in the gait cycle, the visible differences may, therefore, be bigger than the actual difference between the segments of robot and human. Visually the robotic upper leg segment hardly deviated from the upper leg orientation. 

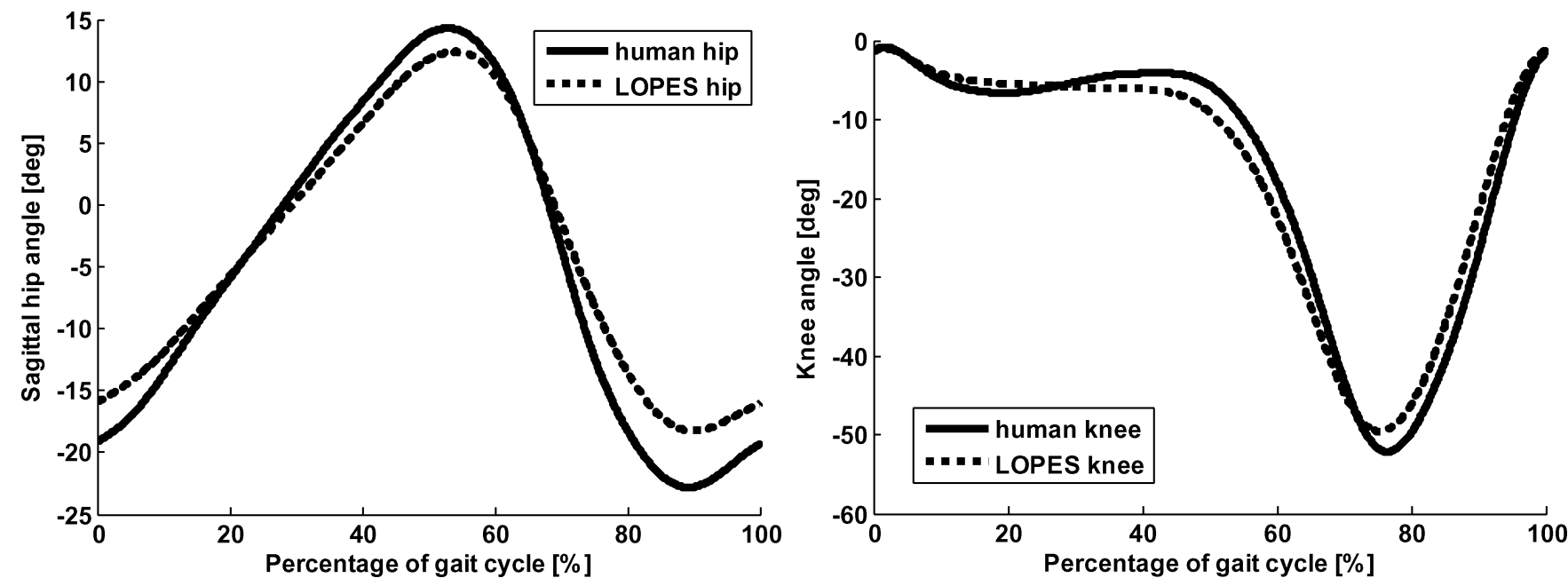

Fig. 7. Graphs of the sagittal hip and knee angles of both human (position tracking measurement with leg-marker-clusters) and robot (joint angle measurements) for several strides during slow walking $(0.75 \mathrm{~m} / \mathrm{s})$.

\section{DISCUSSION}

The prototype of LOPES is fully functional. Until now, about 30 healthy persons have "walked in" the device for about an hour in the "patient in charge" mode without any mechanical problems. (a movie can be found ${ }^{1}$ ). However, for use in clinical research LOPES must be made fully patient safe. This implies, for example, an independent safety circuit that can power the system down in case of any danger and a covering of all possible dangerous moving parts.

The slip that appeared during applying peak torques can be solved by not relying on the friction between cable and disc for force transmission, but by rigidly fixing the cables to this disk. Another design optimization can be made in reducing the weight of the leg parts, as these largely determine the resistive torques while moving the legs. The weight of the legs can be considerably reduced by reducing their dimensions and selecting different materials, and as such are merely a matter of product engineering.

The evaluations showed a decent agreement to the stated demands. Although the resistive torques in the "patient in charge" mode may seem considerable, all people that walked in the LOPES robot reported to experience little to no obstruction to normal walking. This is explained by the relatively slow movements that take place and due to the natural feel of a slight increase of mass. This experience is supported by the EMG measurements, which show only slight differences between walking with and without the LOPES device. Our findings agree with literature on walking with added weight [25].

The differences in the orientation between the human and the robotic limbs appear to be reasonably small, at least for control purpose. A substantial part of the error in hip angle appeared to be caused by secondary rotations of the marker clusters, caused by deformation of the leg due to muscle contractions. A more critical comparison between orientations is recommended for future work in order to judge on the feasibility of the LOPES robot for inverse-dynamics gait measurements. Current measurements indicate that position/angle measurement of the legs

\footnotetext{
${ }^{1}$ http://www.bw.ctw.utwente.nl/research/projects/
}

via the robot device is not sufficiently accurate for inverse dynamic calculations. However, it appeared accurate enough for a safe implementations of an impedance controller that interferes with a walking subject [26].

\section{CONCLUSION}

We designed and evaluated a gait rehabilitation robot prototype that functions as a kinaesthetic (mechanically interactive) interface. It is impedance controlled on eight DOFs and capable of a force bandwidth of $4 \mathrm{~Hz}$ for large forces up to $12 \mathrm{~Hz}$ for smaller forces. Its DOFs allow free leg motions and a free 3-D translation of the pelvis, maintaining the fundamental instability of upright standing and walking. The only possibly important motions that are blocked (except for play) are the pelvis rotations.

The robot is an exoskeleton that moves in parallel with the legs of a person walking on a treadmill, at pelvis height flexibly connected to the fixed world. It allows people to walk unhindered in its "patient in charge mode. It also allows enforcing a gait pattern when configured for its "robot in charge" mode. The actual use will be in between both modes; in its so called "therapist in charge" mode, where selective corrective or supportive torques can be applied to the leg-joints and the pelvis of patients who are walking on their own effort.

Evaluation of the design showed that unhindered walking in the device is very possible, and that any torques/forces needed to impose a gait pattern can be achieved. Also, limb orientations of the robot and the walking subject agree well, sufficient for stable implementation of training and lower level control. Preliminary results of leg muscle EMG measurements show little deviation between treadmill walking and walking with the LOPES exoskeleton. However, any clinical evaluation with patients has yet to be carried out.

\section{ACKNOWLEDGMENT}

The authors would like to thank G.-J. Nevenzel for his technical contribution to the project. 


\section{REFERENCES}

[1] H. Van der Kooij, J. Veneman, and R. Ekkelenkamp, "Design of a compliantly actuated exo-skeleton for an impedance controlled gait trainer robot," in Proc. 28th Annu. Int. Conf. IEEE Eng. Med. Biol. Soc., New York City, Aug. 2006, pp. 189-193.

[2] J. F. Veneman, R. Ekkelenkamp, R. Kruidhof, F. C. T. van der Helm, and H. van der Kooij, "A Series elastic- and bowden-cable-based actuation system for use as torque actuator in exoskeleton-type robots," Int. J. Robot. Res., vol. 25, pp. 261-281, Mar. 1, 2006.

[3] P. Lum, D. Reinkensmeyer, R. Mahoney, W. Z. Rymer, and C. Burgar, "Robotic devices for movement therapy after stroke: Current status and challenges to clinical acceptance," Top Stroke Rehabil., vol. 8, pp. $40-53,2002$.

[4] K. H. Mauritz, "Gait training in hemiparetic stroke patients," Eura Medicophys., vol. 40, pp. 165-178, Sep. 2004.

[5] B. H. Dobkin, "Strategies for stroke rehabilitation," Lancet Neurol., vol. 3, pp. 528-536, Sep. 2004.

[6] S. Hesse, H. Schmidt, C. Werner, and A. Bardeleben, "Upper and lower extremity robotic devices for rehabilitation and for studying motor control," Current Opinion Neurol., vol. 16, pp. 705-710, Dec. 2003.

[7] G. Colombo, M. Joerg, R. Schreier, and V. Dietz, "Treadmill training of paraplegic patients using a robotic orthosis," J. Rehabil. Res. Develop., vol. 37, pp. 693-700, 2000.

[8] D. J. Reinkensmeyer, D. Aoyagi, J. L. Emken, J. A. Galvez, W. Ichinose, G. Kerdanyan, S. Maneekobkunwong, K. Minakata, J. A. Nessler, R. Weber, R. R. Roy, R. de Leon, J. E. Bobrow, S. J. Harkema, and V. R. Edgerton, "Tools for understanding and optimizing robotic gait training," J. Rehabil. Res. Develop., vol. 43, pp. 657-670, Sep.-Oct. 2006.

[9] S. Hesse and D. Uhlenbrock, "A mechanized gait trainer for restoration of gait," J. Rehabil. Res. Develop., vol. 37, pp. 701-708, 2000.

[10] H. Schmidt, C. Werner, R. Bernhardt, S. Hesse, and J. Kruger, "Gait rehabilitation machines based on programmable footplates," J. Neuroeng. Rehabil., vol. 4, p. 2, 2007.

[11] G. Kwakkel, R. C. Wagenaar, J. W. Twisk, G. J. Lankhorst, and J. C. Koetsier, "Intensity of leg and arm training after primary middle-cerebral-artery stroke: A randomised trial," Lancet, vol. 354, pp. 191-196, 1999.

[12] M. Lotze, C. Braun, N. Birbaumer, S. Anders, and L. G. Cohen, "Motor learning elicited by voluntary drive," Brain, vol. 126, pp. 866-872, Apr. 2003.

[13] N. A. Bayona, J. Bitensky, K. Salter, and R. Teasell, "The role of taskspecific training in rehabilitation therapies," Topics Stroke Rehabil., vol. 12, pp. 58-65, 2005.

[14] L. Nilsson, J. Carlsson, A. Danielsson, A. Fugl-Meyer, K. Hellstrom, L. Kristensen, B. Sjolund, K. S. Sunnerhagen, and G. Grimby, "Walking training of patients with hemiparesis at an early stage after stroke: A comparison of walking training on a treadmill with body weight support and walking training on the ground," Clin. Rehabil., vol. 15, pp. 515-527, 2001.

[15] N. Hogan, H. I. Krebs, B. Rohrer, J. J. Palazzolo, L. Dipietro, S. E. Fasoli, J. Stein, R. Hughes, W. R. Frontera, D. Lynch, and B. T. Volpe, "Motions or muscles? Some behavioral factors underlying robotic assistance of motor recovery," J. Rehabil. Res. Develop., vol. 43, pp. 605-618, Sep.-Oct. 2006.

[16] R. Riener, L. Lunenburger, and G. Colombo, "Human-centered robotics applied to gait training and assessment," J. Rehabil. Res. Develop., vol. 43, pp. 679-694, Sep.-Oct. 2006.

[17] R. Q. V. D. Linde and P. Lammertse, "HapticMaster-A generic force controlled robot for human interaction," Industrial Robot, vol. 30, pp. 515-524, 2003.
[18] H. van der Kooij, J. Veneman, and R. Ekkelenkamp, "Compliant actuation of exoskeletons," in Mobile Robotics-Towards New Applications, A. Lazinica, Ed. Mammendorf, Germany: Verlag Rober Mayer-Scholz, 2006.

[19] R. Ekkelenkamp, J. Veneman, and H. van der Kooij, "LOPES: Selective control of gait functions during the gait rehabilitation of CVA patients," in 9th Int. Conf. Rehabil. Robot., Chicago, IL, 2005, pp. 361-364.

[20] J. F. Israel, D. D. Campbell, J. H. Kahn, and T. G. Hornby, "Metabolic costs and muscle activity patterns during robotic- and therapist-assisted treadmill walking in individuals with incomplete spinal cord injury," Physical Therapy, vol. 86, pp. 1466-1478, Nov. 2006.

[21] D. P. Ferris, J. M. Czerniecki, and B. Hannaford, "An ankle-foot orthosis powered by artificial pneumatic muscles," J. Appl. Biomech., vol. 21, pp. 189-197, May 2005.

[22] H. I. Krebs and N. Hogan, "Therapeutic robotics: A technology push," Proc. IEEE, vol. 94, no. 9, pp. 1727-1738, Sep. 2006.

[23] D. A. Winter, Biomechanics and Motor Control of Human Movement, 2nd ed. New York: Wiley, 1990.

[24] J. A. Galvez, G. Kerdanyan, S. Maneekobkunwong, R. Weber, M. Scott, S. J. Harkema, and D. J. Reinkensmeyer, "Measuring human trainers' skill for the design of better robot control algorithms for gait training after spinal cord injury," in 9th Int. Conf. Rehabil. Robotics, 2005, pp. 231-234.

[25] E. H. F. Van Asseldonk, R. Ekkelenkamp, J. F. Veneman, F. C. T. Van der Helm, and H. Van der Kooij, "Selective control of a subtask of walking in a robotic gait trainer(LOPES)," presented at the 10th Int. Congress Rehabil. Robotics, Noordwijk aan Zee, 2007.

[26] J. W. Noble and S. D. Prentice, "Adaptation to unilateral change in lower limb mechanical properties during human walking," Exp. Brain Res., vol. 169, pp. 482-95, Mar. 2006.

Jan F. Veneman, photograph and biography not available at the time of publication.

Rik Kruidhof, photograph and biography not available at the time of publication.

Edsko E. G. Hekman, photograph and biography not available at the time of publication.

Ralf Ekkelenkamp, photograph and biography not available at the time of publication.

Edwin H. F. Van Asseldonk, photograph and biography not available at the time of publication.

Herman van der Kooij, photograph and biography not available at the time of publication. 